\title{
Key Technology of Electrochemical Micromachining with Laser masking
}

\author{
Li Xiaohai ${ }^{1, a^{*}}$, Wang Shuming ${ }^{2, b}$, Wang Dong ${ }^{1, c}$, Tong Han ${ }^{1, d}$ \\ ${ }^{1}$ School of Mechanical Engineering, Jiamusi University,Jiamusi, Heilongjiang, China \\ ${ }^{2}$ School of Materials Science and Engineering, Jiamusi University, Jiamusi, Heilongjiang, China \\ alixh@jmsu.edu.cn, ${ }^{b}$ wsmwsm2000@163.com, ${ }^{c}$ wd921120@126.com and ${ }^{\text {d1921099967@qq.com }}$
}

Keywords: Electrochemical micromachining, Fiber laser, Masking, 304 Stainless steel, Combined machining.

Abstract. In order to fabricate the micro patterns cavities with micro size on 304 stainless steel, electrochemical micromachining (EMM) with laser masking based on surface modification by fiber laser masking was studied, and a device of laser-assisted EMM with the special pulse power supply was developed to achieve micro machining, which possessed the complete control system. Laser marking on the surface of 304 stainless steel can first be realized by fiber laser scanning and heating. During laser marking, metal oxide layer with pre-designed pattern can be formed by laser marking, and phase transformation can also occur at the laser scanning area on 304 stainless steel surface, which forms the laser masking layer with good corrosion resistance. The stainless steel surface with laser masking layer is subsequently etched by EMM, the laser masking layer severs as the temporary protective layer without relying on lithography mask. Accordingly, micro pattern cavities can fast be fabricated. Through machining experiments, the typical samples $23 \mu \mathrm{m}$ deep were fabricated under the optimized parameters.

\section{Introduction}

The methods of non-traditional machining are very effective to fabricate small metal parts, especially electrochemical machining and laser beam machining, which are widely studied by many scholars. For example, Schuster [1] proposed the EMM with nanosecond ultra-short pulse current, which can achieve the sub micron precision. Di Zhu [2] adopted the reciprocating wire to cut the micro thin-wall by using micro wire electrochemical cutting with nanosecond pulse current; Shufeng Sun[3] machined high precision micro gear with femtosecond laser. But laser processing belongs to the heat processing by using photothermal effect, and metal surface scanned by laser beam have the heat affected layer and easy deformation. Despite there is no heat affected layer formed during EMM, it is also difficult to guarantee the shape accuracy and the dimension of machined parts owing to the existence of stray corrosion of EMM. Now many scholars try to combine electrochemical machining with laser processing, such as Chaoyang Zhang [4] used conductive transparent ITO glass as cathode for EMM, the passivation film formed during EMM was removed by laser radiation, and then electrolysis would continue and fabricate micro structures. Pajak [5] fabricated micro parts by using electrolyte jet with the laser-assisted heating, which have achieved good processing effect.

In this paper, by combining EMM with laser masking, a new device with the special pulse power supply was developed to fabricate the micro-pattern cavities on the surface of 304 stainless steel, which was equipped with the complete control system to fully realize micromachining. The protective masking layer was first made by laser marking, and then the metal of the unmasked area is removed to fast fabricate the micro pattern cavities with micro size. This method possesses very significant development in micromachining field.

\section{The development of the device of EMM with laser masking.}

In order to study the mechanism of EMM with laser masking, a suit of EMM device with laser masking was developed, as shown in Fig. 1. The control system for EMM with laser masking is shown in Fig.2.Pulsed fiber laser had been selected for its excellent beam quality, long maintenance free time, 
high efficiency, high pulse frequency, no need of water cooling and low operating cost. IPG pulsed fiber laser made in Germany is used to provide energy for the laser masking. The optical system is SCANLAB galvanometer made in Germany. The power of fiber laser are adjustable in the range of $0-20 \mathrm{~W}$, the minimum laser beam diameter is $10 \mu \mathrm{m}$, the frequency is adjustable between $0-100 \mathrm{kHz}$, the laser wavelength is $1.064 \mu \mathrm{m}$, and high repetitive positioning precision is less than $2 \mu \mathrm{m}$. Granite has the advantages of high hardness, wear resistance, good vibration resistance, good acid-resistant and alkali-resistant, no internal residual stress, low thermal expansion coefficient, and granite was adopted as the machine bed.The three-axis table with the resolution $0.4 \mu \mathrm{m}$ makes $\mathrm{X}$-axis, Y-axis and Z-axis feed. The surface modification module by using fiber laser and electrochemical machining system are installed on the $\mathrm{Z}$ axis to solve the positioning problem of laser masking and EMM. The EMM system is composed of electrolyte circulating system and micro pulse power supply for EMM.The electrolyte circulating system provides fresh electrolyte for EMM, including a tank, pipeline, electrolyte tank, nozzle, micro diaphragm pumps, control valves, filters and other components. $1.8 \mathrm{~mol} / \mathrm{L}$ concentration of $\mathrm{NaNO}_{3}$ passivation electrolyte was selected. The tool electrode is the stainless steel sheet with simple shape, and workpiece is the 304 stainless steel masked by fiber laser with $1 \mathrm{~mm}$ thick.

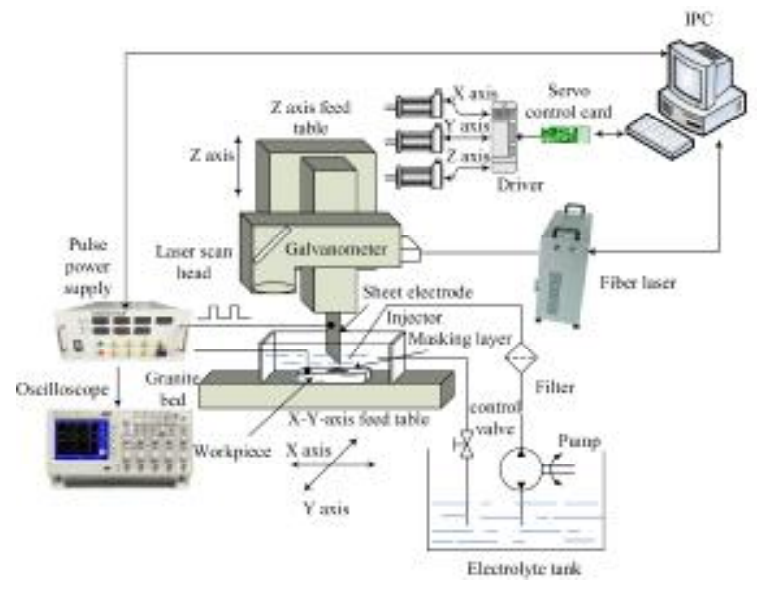

Fig.1 The developed device for EMM combining fiber laser masking

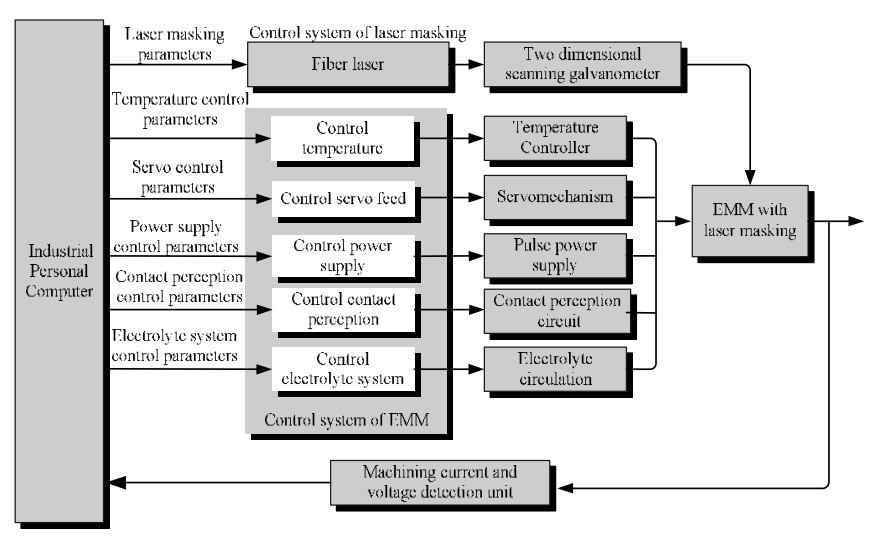

Fig.2 Structure block diagram of control system for EMM with fiber laser masking

The principle of the power supply diagram is shown in Fig.3, which consists of the following modules: high frequency logic pulse circuit, MOSFET on-off control circuit and a driving pulse amplifying circuit, main circuit, power supply protection circuit, the detecting circuit of machining state, PIC microprocessor and PC serial communication circuit, contact perception contact sensing module. The frequency and duty ratio of the designed pulse power supply for EMM can be adjustable, output is stable and accurate, and the short circuit protection modular is designed to get self-protect performances when short circuit occurs. The high frequency pulse logic generating unit is programmed by the PIC micro controller to generate high frequency logic pulse signals, which are amplified by SN7407 and TPS 2812 to drive MOSFET IRF840 through the power amplifier module. The machining voltage output can continuously be adjusted by auto-transformer, and a continuous voltage regulation and a rectification filtering circuit can convert the power frequency alternating current into the direct current, which is controlled by MOSFET tube to provide the required pulse voltage for EMM. PIC micro controller through serial ports transfers information with industrial control computer and set processing parameters. When short circuit occurs during EMM, a short circuit protection circuit can detect the occurrence of short circuit through the current sensor, protects the power supply. Then the short circuit signal is transferred to industrial control computer, industrial control computer gives instructions to raise the electrode and eliminate the short circuit. The optocoupler isolation devices are adopted in interface circuit to insulate strong current and weak current isolation, and protect the power supply of EMM. A contact perception module was design in a power supply for EMM to determine a reasonable gap between tool electrode and workpiece. 


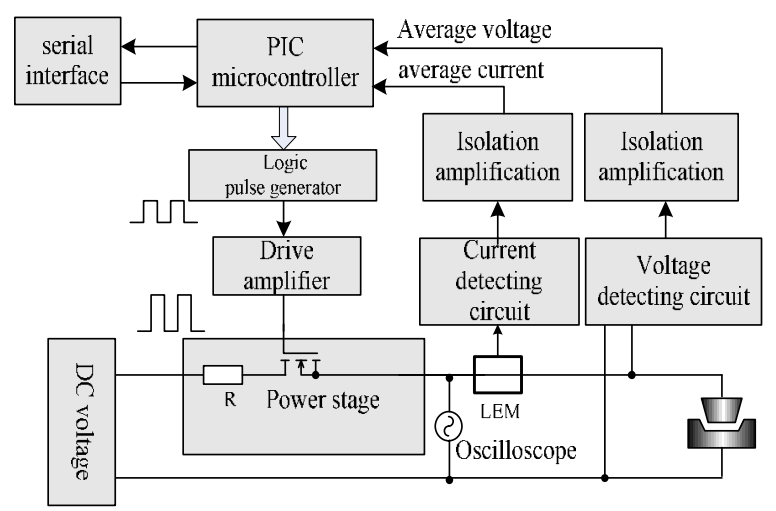

Fig.3 Principle diagram of micro ECM pulse power supply

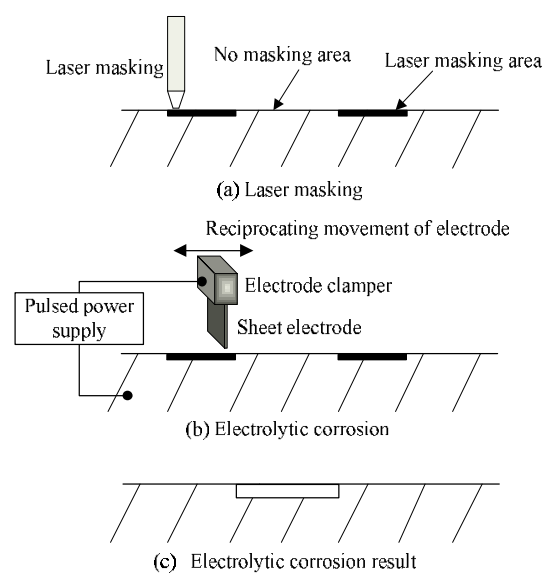

Fig.4 Schematic diagram of EMM with laser masking

\section{Mechanism of EMM with laser masking}

Principle of EMM with laser masking Through processing experiments, the mechanism of EMM with laser masking was studied on the developed device. The surface of 304 stainless steel is scanned by the laser beam according to the planned route to generates the certain protective masking patterns. The specific process is shown as Fig.4. By using precision fiber laser, the three-coordinate feeding table with high resolution, galvanometer and optical lens, the laser focus is controlled to scan the surface of the workpiece by laser direct writing, and then laser masking pattern can be generate by the NC system, as shown in Fig.4 (a). Then, EMM with high frequency pulse current is adopted to fabricate micro structure, cathode is sheet electrode of stainless steel, 304 stainless steel with laser masking patterns serves as anode. Before EMM, the cathode position relative to anode must be adjusted to ensure there is a certain distance between two electrodes. The sheet electrode repeatedly scans above the surface of the workpiece. Owing to the existence of laser masking layer, the dissolve rate of masked metal area is obviously slower than that of the unmasked metal area. The masking layer plays a temporary protective effect, the localized machining can be realized, as shown in Fig. 4 (b). Through EMM, the masked area is protected by a certain mask pattern to form a two-dimensional micro cavity, as shown in Fig.4 (c).The etched depth of one time EMM with laser masking is very small, so by repeating the above processes many times, the etched depth can remarkably increase.

$X$ ray diffraction analysis of laser masking layer. Mechanism analysis of corrosion resistance of laser masking layer was analyzed based on X ray diffraction analysis (XRD). First, the surface of 304 stainless steel was scanned by laser marking to form recast layer, namely laser masking layer. The processing conditions of forming laser masking layer were that laser power is $5 \mathrm{~W}$, laser scanning speed is $20 \mathrm{~mm} / \mathrm{s}$, pulse frequency is $80 \mathrm{kHz}$ and line spacing is $10 \mu \mathrm{m}$. Then, the components of the marked surface of the 304 stainless steel after laser masking and the components of the unmarked surface of 304 stainless steel without laser masking were compared by XRD, as shown in Fig.5. Cr, Fe and other metal oxides have appeared on the marked surface of 304 stainless steel after fiber laser masking, which is caused by oxygen oxidizing reaction in the air to form a sub micron thickness metal oxide layer. During EMM in $\mathrm{NaNO}_{3}$ electrolyte, most of electrons losing at the anodic masking area were consumed in oxygen evolution, and $\mathrm{Cr}$, Fe oxide layer generated on the 304 stainless steel surface hinders metal dissolution and greatly reduces the electrolytic removal rate in EMM, so the dissolution rate of masked metal surface is lower than that of unmasked metal surface. Consequently, the laser masking layer produced by laser masking plays a most important role in selectively dissolving metals, which can realize localized machining. At the same time, laser masking also cause phase transition on the surface of 304 stainless steel. From Fig.5, austenite $\gamma$-Fe phase increased by laser masking, which can improve the corrosion resistance of stainless steel and then the speed of dissolution can be reduced. 


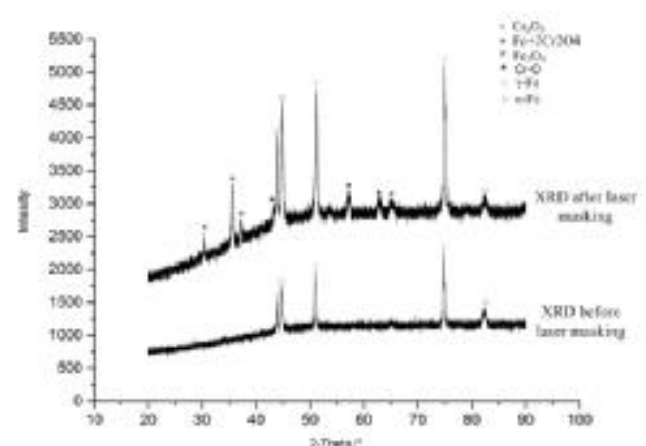

Fig.5 XRD analysis of 304 stainless steel matrix and laser masking layer

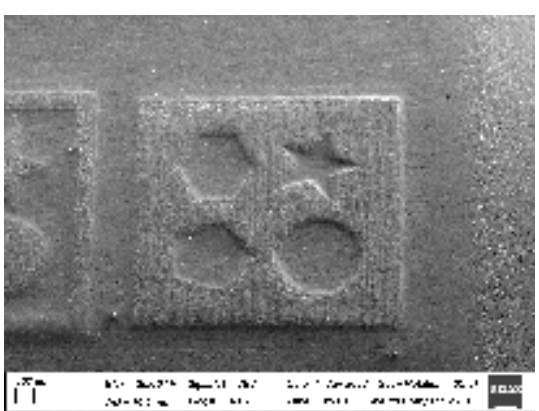

Fig.6 Micro cavities fabricated by EMM with laser masking

\section{Experiment of EMM with Laser Masking}

By optimizing machining paremeters, the typical samples were fabricated. Surface modification of 304 stainless steel is first treated by laser masking when laser power is $5 \mathrm{~W}$, laser scanning speed is $20 \mathrm{~mm} / \mathrm{s}$, the laser beam diameter is $50 \mu \mathrm{m}$ and line spacing is $10 \mu \mathrm{m}$. The EMM parameters are that machining voltage is $9.5 \mathrm{~V}, \mathrm{NaNO}_{3}$ electrolyte concentration is $1.8 \mathrm{~mol} / \mathrm{L}$, inter-electrode gap is $220 \mu \mathrm{m}$, duty cycle of pulse power supply is $1: 16$ and pulse on time is $50 \mu \mathrm{s}$. This processing has good prospect for fabricating micro channels, micro patterns and micro mould cavities and so on. Fig.6 is the samples fabricated through EMM with laser masking three times, and the cavity depth is about $23 \mu \mathrm{m}$, the scanning speed of the laser masking is $20 \mathrm{~mm} / \mathrm{s}$. Many samples can simultaneously be fabricated, and then the processing efficiency is very high.

\section{Conclusions}

The new device of EMM with laser masking was developed, which possessed the complete control system. By fiber laser marking, Fe and Cr oxide masking layer can be generated on 304 stainless steel surface. The laser masking can make the dissolution rate of no-machining area covered by Fe oxide and $\mathrm{Cr}$ oxide lower than that of machining area without laser masking, and then localization of EMM are improved. Through experiments, the typical cavities with $23 \mu \mathrm{m}$ depth were fabricated, which proved that the developed device of EMM with laser masking is feasible in micro machining.

\section{Acknowledgements}

This work was financially supported by National Natural Science Foundation of China(51375208).

\section{References}

[1] M. KOCK, V. KIRCHNER, R. SCHUSTER, Electrochemical micromachining with ultrashort voltage pulses versatile method with lithographical precision, Electrochimica Acta. 48(2003)3213-3219

[2] Q. YU, D. ZHU, Y.B. ZENG, et al, Wire Electrode Micro-electrochemical Machining with Tool Micro-amplitude Reciprocating Motion, Acta Aeronautica ET Astronautica Sinica. 33(2012)1-8

[3] S.F. SUN, P.P. WANG, W. XUE, Fabrication technique research on micro gear based on two-photo of femtosecond laser, Journal of Mechanical Engineering. 47(2011)193-198

[4] C.Y. ZHANG, C.L. QIN, Q.C. FENG, et al, Investigation on Localisation and Three-dimensional Micro-etching Based on Pulse Laser Electrochemical Machining, Journal of Mechanical Engineering. 50(2014)200-208 
[5] P. T. PAJAK, V.A. DESIL, D. K. HARRISON, et al, Precision and efficiency of laser assisted jet electrochemical machining, Precision Engineering. 30(2006)288-298 